Elsevier Editorial System(tm) for Computer Methods and Programs in Biomedicine Manuscript Draft

Manuscript Number:

Title: TUBERCULINE REACTION MEASURED BY INFRARED THERMOGRAPHY

Article Type: Full Length Article

Section/Category: Section II: Systems and Programs

Keywords: Tuberculine test; Infrared Thermography; Image processing

Corresponding Author: Prof. Marcos Faundez-Zanuy,

Corresponding Author's Institution: Fundació Tecnocampus

First Author: José Antonio Fiz, PhD

Order of Authors: José Antonio Fiz, PhD; Manuel Lozano; Enrique Monte-Moreno, PhD; Adela GonzalezMartinez; Laura Rodriguez-Pons; Caroline Becker; Marcos Faundez-Zanuy; Juan Ruiz-Manzano, PhD

Abstract: Setting: The infection with Mycobacterium tuberculosis gives a delayed immune response, measured by the tuberculine skin test. We present a new technique for evaluation based on automatic detection and measurement of skin infrared emission.

Design: 21 subjects $(57.0 \pm 17.8 \mathrm{yr}),(7 / 14, \mathrm{M} / \mathrm{F})$ with suspected tuberculosis contact were examined with an IR thermal camera, 48 hours after skin injection.

Results: In 15 subjects, IR analysis was positive for tuberculine test. Mean temperature of injection area was higher, around $1{ }^{\circ} \mathrm{C}$, for the positive group $\left(36.0 \pm 0.3{ }^{\circ} \mathrm{C}\right.$ positive group; $35.2 \pm 1.7{ }^{\circ} \mathrm{C}$ negative group, $\mathrm{p}<0.001$, Non parametric U Mann-Whitney Test).

Conclusion: IR may represent an improved estimation of tuberculosis infection, given that it does not depend on reader variability and measures the increase of heat irradiation produced by the allergic tuberculine response.

Suggested Reviewers: Jiri Mekyska PhD

Associate Professor, Brno University of Technology (Czech Republic)

j.mekyska@gmail.com

Expert in biomedical applications and thermal imaging

Carlo Morabito PhD

vice-rector, University Reggio Calabria (Italy)

morabito@unirc.it

Expert in soft computing systems and biomedical applications

Anna Esposito PhD

professor, University of Naples

Anna.ESPOSITO@unina2.it

Expert in computer science and thermal imaging

Jordi Sole-Casals PhD 
Associate Professor, University of Vic

jordi.sole@uvic.cat

Expert in biomedical applications and signal processing

Opposed Reviewers: 
We declare no conflict of interests.

The authors

$25 / 2 / 2015$ 


\title{
TUBERCULINE REACTION MEASURED BY INFRARED THERMOGRAPHY
}

\author{
José Antonio Fiz ${ }^{1,3^{*}}$ (jafiz@msn.com) (JAF) MD, PhD \\ Manuel Lozano ${ }^{2}$ (mlozanogar@gmail.com) $\quad(M L) \quad$ Engineer \\ Enrique Monte-Moreno ${ }^{3}$ (enric.monte@upc.edu) (EM) Engineer, PhD \\ Adela Gonzalez-Martinez ${ }^{1}$ (agonzalezm.germanstrias@gencat.cat) (AG) Technician \\ Laura Rodriguez Pons ${ }^{1}$ (laurarodriguezpons@gmail.com) (LR) MD \\ Caroline Becker ${ }^{1}$ (arialcb@gmail.com) (CB) MD \\ Marcos Faundez-Zanuy ${ }^{4}$ (faundez@tecnocampus.cat) (MF) Engineer, PhD \\ Juan Ruiz Manzano ${ }^{1}$ (jruizmanzano.germanstrias@gencat.cat)(JR) MD, PhD
}

${ }^{1}$ Pulmonology Department, Germans Trias I Pujol Universitary Hospital. $8^{\text {th }}$, Carretera del Canyet s/n. 08916, Badalona, Spain. Phone Number

${ }^{2}$ Innovation Group, Health Sciences Research Institute of Germans Trias i Pujol Fundation(IGTIP). Ctra, de Can Ruti /Camí de les escoles s/n, 08916, Badalona, Spain.

${ }^{3}$ Center for Language and Speech Technologies and Applications (TALP), Technical University of Catalunya (UPC), Jordi Girona, 1-3, S112, Campus Nord, Barcelona, Spain.

${ }^{4}$ Escola Superior Politècnica Tecnocampus Mataró. Spain Phone Number: 34931696501

Running head: Tuberculine test measured by Infrared Thermography

Word Count: 3046

Keywords: Tuberculine test, Infrared Thermography

Corresponding author: Dr José Antonio Fiz 
Setting: The infection with Mycobacterium tuberculosis gives a delayed immune response, measured by the tuberculine skin test. We present a new technique for evaluation based on automatic detection and measurement of skin infrared emission.

Design: 21 subjects $(57.0 \pm 17.8 \mathrm{yr}),(7 / 14, \mathrm{M} / \mathrm{F})$ with suspected tuberculosis contact were examined with an IR thermal camera, 48 hours after skin injection.

Results: In 15 subjects, IR analysis was positive for tuberculine test. Mean temperature of injection area was higher, around $1{ }^{\circ} \mathrm{C}$, for the positive group $(36.0 \pm 0.3$ ${ }^{\circ} \mathrm{C}$ positive group; $35.2 \pm 1.7{ }^{\circ} \mathrm{C}$ negative group, $\mathrm{p}<0.001$, Non parametric U MannWhitney Test).

Conclusion: IR may represent an improved estimation of tuberculosis infection, given that it does not depend on reader variability and measures the increase of heat irradiation produced by the allergic tuberculine response. 
${ }^{*}$ Highlights (for review)

\section{Highlights}

- First paper devoted to tuberculine reaction measurement based on thermal imaging.

- Proposed method alleviates the variability of a human supervisor

- Low cost system for solving a challenging diagnose 


\section{TUBERCULINE REACTION MEASURED BY INFRARED THERMOGRAPHY}

3

4

5

6

7
José Antonio Fiz ${ }^{1,3^{*}}$ (jafiz@msn.com) (JAF)

Manuel Lozano² (mlozanogar@gmail.com) (ML)

Enrique Monte-Moreno ${ }^{3}$ (enric.monte@upc.edu) (EM)

Adela Gonzalez-Martinez ${ }^{1}$ (agonzalezm.germanstrias@gencat.cat)

Laura Rodriguez Pons ${ }^{1}$ (laurarodriguezpons@gmail.com) (LR)

Caroline Becker ${ }^{1}$ (arialcb@gmail.com) (CB)

Marcos Faundez-Zanuy (faundez@tecnocampus.cat) (MF)

Juan Ruiz Manzano (iruizmanzano.germanstrias@gencat.cat) (JR)
MD, PhD

Engineer

Engineer, PhD

(AG) Technician

MD

MD

Engineer, PhD

MD, PhD

\footnotetext{
${ }^{1}$ Pulmonology Department, Germans Trias I Pujol Universitary Hospital. $8^{\text {th }}$, Carretera del
} Canyet s/n. 08916, Badalona, Spain. Phone Number

${ }^{2}$ Innovation Group, Health Sciences Research Institute of Germans Trias i Pujol Fundation(IGTIP). Ctra, de Can Ruti /Camí de les escoles s/n, 08916, Badalona, Spain.

${ }^{3}$ Center for Language and Speech Technologies and Applications (TALP), Technical University of Catalunya (UPC), Jordi Girona, 1-3, S112, Campus Nord, Barcelona, Spain.

${ }^{4}$ Escola Superior Politècnica Tecnocampus Mataró. Spain Phone Number: 34931696501

Running head: Tuberculine test measured by Infrared Thermography

Word Count: 3046

Keywords: Tuberculine test, Infrared Thermography 


\section{ABSTRACT}

32

Setting: The infection with Mycobacterium tuberculosis gives a delayed immune response, measured by the tuberculine skin test. We present a new technique for evaluation based on automatic detection and measurement of skin infrared emission.

Design: 21 subjects $(57.0 \pm 17.8 \mathrm{yr}),(7 / 14, \mathrm{M} / \mathrm{F})$ with suspected tuberculosis contact were examined with an IR thermal camera, 48 hours after skin injection.

Results: In 15 subjects, IR analysis was positive for tuberculine test. Mean temperature of injection area was higher, around $1{ }^{\circ} \mathrm{C}$, for the positive group $(36.0 \pm 0.3$ ${ }^{\circ} \mathrm{C}$ positive group; $35.2 \pm 1.7{ }^{\circ} \mathrm{C}$ negative group, $\mathrm{p}<0.001$, Non parametric $\mathrm{U}$ MannWhitney Test).

Conclusion: IR may represent an improved estimation of tuberculosis infection, given that it does not depend on reader variability and measures the increase of heat irradiation produced by the allergic tuberculine response. 


\section{INTRODUCTION}

According to the World Health Organization, two billion people are infected with the tuberculosis (TB) bacilli ${ }^{1}$. It is estimated that every year eight million new cases appear. TB is a contagious disease not totally under control with great differences between rich and poor countries ${ }^{2}$. The diagnosis of TB is based on the interpretation of a skin immunologic reaction. The infection with Mycobacterium tuberculosis gives a delayed immune response, measured by the tuberculin skin test (TST) ${ }^{3}$. This consists of the intradermal injection of tuberculin purified protein derivative (PPD) and measurement of the resulting reaction. The induration size, measured in millimeters, indicates if the test result is negative or positive. Palpation and pen methods have been typically applied to measure the PPD reaction, but the measurement depends on the subjective interpretation of the reaction area ${ }^{4,5}$. New technologies are currently being developed to improve the sensitivity and specificity of the TB diagnosis ${ }^{6}$.

In this study, we present a new technique for the evaluation of the tuberculine reaction based on the automatic detection and measurement of infrared (IR) emission of inflammatory effects produced by tuberculine immune response. IR thermal imaging is a noninvasive technique for monitoring temperatures and is widely applied in medicine $\mathrm{e}^{7,8}$. The IR radiation increases due to inflammatory processes, such as that in the tuberculine reaction. An increased IR radiation is most likely caused by elevated blood flow, metabolic activity, and inflammatory reactions ${ }^{9}$. Therefore IR thermal imaging can be used to measure the rise in temperature due to an inflammatory process, which causes the increase of the blood flow with vascular dilatation, blood proteins and cellular extravasations ${ }^{10}$.PPD inflammatory response is characterized by edema, leukocyte lymphocyte-mononuclear infiltration, and erythrocyte extravasation in the dermis and epidermis ${ }^{11}$. Our hypothesis in the present work was that intradermal 
77 inflammatory PPD reaction produced an increase in the temperature of the injected area,

1

78 which could be measured by IR thermal imaging, and the features extracted from the

79 resulting IR images after applying digital image processing techniques, helped to 80 improve the reliability in the TB diagnosis. 


\section{Ethics Statement}

The study was conducted in the Respiratory Function Laboratory at HUGTIP, from February- September 2014, and approved by the hospital's Human Research and Ethics Committee. All participants came from pulmonology dept to discard tuberculosis contact and gave written informed consent, following the World Medical Association's Declaration of Helsinki on Ethical Principles for Medical Research Involving Human Subjects.

\section{Mantoux tuberculin skin test}

The Mantoux tuberculin skin test (TST) was performed in all subjects by intradermal injection of $0.1 \mathrm{ml}$ of tuberculine purified protein derivative (PPD) (Tuberculina PPD Evans 2 UT/0.1 ml, UCB Pharma S.A., Madrid, Spain) in the anterior forearm. First, the reading of the Mantoux TST was manually performed 48 hours following the skin injection ${ }^{3}$ by inspection, palpation, and measurement of the induration. An induration size greater than $0.5 \mathrm{~cm}$ was considered to be a positive TST result. A technician with more than 20 years experience in TST lecture has made all TST test ${ }^{12}$.

\section{IR thermal imaging}

The size of the induration was also measured by IR thermal imaging. Subjects remained in the laboratory room for at least 15 minutes to achieve thermal equilibrium. Temperature, humidity and air circulation were all controlled. Laboratory temperature was maintained between $23 \pm 1{ }^{\circ} \mathrm{C}$, and humidity was around $50 \%$. Subjects were requested not to consume hot drinks or food for at least an hour before image session, nor use any skin preparations such as creams or talcum powder. Emissivity of the skin 
106

107

108

109

110

111

112

113

114

115

116

117

118

119

120

121

122

123

124

125

126

127

128

was assumed at a value of $0.98 \pm 0.01$. There was no ceiling air filter. The lighting in the laboratory was maintained at wavelengths longer than 1 micron.

The IR thermal images were obtained from the anterior surface of the forearm of each subject, using a TiR32 Fluke Camera (2009 Fluke Corporation USA; 320x240 Focal plane array; $0.01 \mathrm{C}^{\mathrm{o}}$ Noise Equivalent Temperature Difference; $8-14 \mu \mathrm{m}$ spectral range). The IR thermal camera was installed on a tripod with a fluke compact photomovie. The distance between the camera focus and the forearm was $40 \mathrm{~cm}$. The forearm was placed on a black foam cushion over a wooden table, with the anterior surface facing the camera focus. To have a reference distance, we placed a $24 \mathrm{~mm}$ diameter coin below the area to be measured. The detection of that reference object allowed us to convert pixels to millimeters (Figure 1). The image acquisition and storage were made with the SmartView® software. Each image was exported in JPG format. Moreover, the temperature measurements were transferred to a text file.

\section{IR image processing}

The IR image processing was performed using Matlab 2014b and following the scheme shown in Figure 2. Each text file containing the temperature data of an IR image was imported in a 2D numerical array (temp). As IR images had different temperature ranges, each temp array was scaled, as in (1), in order to have values between 0 and 1 , thus obtaining the gray scale image temp_n (Figure 3-a).

$$
\text { temp_n } n=\frac{t e m p-T_{\min }}{T_{\max }-T_{\min }}
$$

$T_{\min }$ and $T_{\max }$ are the minimum and the maximum temperatures in temp respectively. After obtaining the gray scale image temp_n, we detected the reference object of known size that was present in the image in order to obtain the conversion 
129 factor from pixels to millimeters. For that purpose, we first applied the Canny edge 130 detector to the normalized image temp_n (Figure 3-b). Then, since the reference object 131 was a circle, we used the circular Hough transform to detect its position and match its 132 edge (note that the Hough transform can be adapted to detect specific shapes in an 133 image $)^{13}$. As the radius in millimeters of the reference object was known, we were able 134 to calculate the conversion factor to express measured lengths in millimeters (Figure 3$135 \mathrm{c})$.

The temperature in the forearm area was much higher than the temperature outside the forearm area (air). Therefore, the values of all pixels in the forearm area were in the upper range of the scaled temp_ $n$ image. In order to clarify the differences between the internal pixels of the forearm area, we applied an intensity transformation to temp_n. All pixels below 0.5 were set at 0 , whereas pixels in the range $0.5-1$ were 141 expanded to the range $0-1$, thus obtaining the temp_e image (Figure 3-d). The difference 142 between the PPD reaction area and the rest of the forearm was more noticeable in temp_e than in temp_n.

The segmentation algorithm searched two different regions: region 1 (PPD reaction area) and region 2 (erythema). A region growing method was used for the 146 image segmentation. Assuming that the PPD reaction area (region 1) was near the 147 image center, the first seed pixel was the pixel with the maximum temperature $\left(\mathrm{P}_{\mathrm{Tmax}}\right)$ 148 inside a centered rectangle area. A binarization was applied to the image temp_e. Pixels 149 of the surface of the forearm were retained (set at 1) and pixels outside the forearm (air) 150 were rejected (set at 0 ), thus obtaining the temp_b binary image (Figure 3 -e). Then, a $1515 \times 5 \mathrm{~cm}$ square, which was centered at $\mathrm{P}_{\mathrm{Tmax}}$, was selected as the analysis area mask. 
Having found the first seed point and defined the analysis area, the region

153 growing algorithm was applied to temp_e by means of the following iterative procedure

until convergence to a fixed point was reached. At each iteration, new sub-regions 1 and

2 were grown from a new seed point, by adding in neighboring pixels according to each sub-region membership criterion. First, sub-region 1 was grown by adding in neighboring pixels that met the criterion in (2).

$$
-A * T h 1<\min S_{1}(i, j)<T h 1 \rightarrow S_{1}(i, j)=r_{e} g_{-} \text {mean } 1-t e m p \_e(i, j)
$$

The parameter reg_mean ${ }_{1}$ was the mean value of all pixels included in the whole membership criterion of a neighboring pixel, Thl was the threshold used to either include or not a neighboring pixel in sub-region 1 , and $A$ was the scale factor of $T h 1$ to define the lower limit of $S_{l}(i, j)$. When there were not neighboring pixels meeting the criterion defined in (2), a new criterion, as defined in (3), was used to add in neighboring pixels to sub-region 2 .

$$
\min \left(\left|S_{2}(i, j)\right|\right)<T h 2 \rightarrow S_{2}(i, j)=\text { reg_mean }_{2}-\text { temp_e}_{-}(i, j)
$$

The parameter reg_mean 2 was the mean value of all pixels included in the whole region 2 , temp_e $(i, j)$ was the intensity value of a neighboring pixel, $S_{2}(i, j)$ was the membership criterion of a neighboring pixel, and Th2 was the threshold used to either include or not a neighboring pixel in sub-region 2 . When no neighboring pixels met the criterion defined in (3), a new seed point was selected and a new iteration started. Each new seed point had to meet the criterion defined in (2) and be at a maximum distance of $15 \mathrm{~mm}$ from the sub-region 1 obtained in the first iteration. 
After the region growing process finished, when no new seed points existed,

175 regions 1 and 2 were formed by a set of sub-regions 1 and 2, respectively (Figure 3-f).

176 The maximum length of both region 1 and region 2 were calculated as the sum of the

177 maximum length of their corresponding sub-regions (Figures 3-g and 3-h). The

178 background area was calculated by removing the pixels belonging to either region 1 or

179 region 2 from the analysis area mask. Then, the area $\left(\mathrm{mm}^{2}\right)$, the minimum, the 180 maximum, the mean, and the standard deviation temperatures $\left({ }^{\circ} \mathrm{C}\right)$ were calculated 181 either for region 1 , region 2, and the background.

Finally, the region 2 was merged with the background if the following criterion

where $t_{\text {meank }}$ and $t_{\text {stdk }}(k=2,3)$ were the mean and standard deviation temperatures of region 2 and background, respectively. Furthermore, region 1 was merged with region 2 and the background if criterion defined in (4) and the following criteria were met: negative/ positive segmentation indicated an IR negative/ positive result respectively. Also, for an expert in IR imaging that made all lectures of patient IR image, the non where $t_{\text {meank }}$ and $t_{\text {stdk }}(\mathrm{k}=1,23,2$, or 3$)$ were the mean and standard deviation temperatures of region 1, region 2+background, region 2, and background, respectively. In those cases, only a background area was obtained as the final result of the image segmentation. Therefore, no reaction area was found and the test was negative. A was met:

$$
t_{\text {mean2 } 2}-t_{\text {std2 }}-t_{\text {mean } 3}-t_{\text {std } 3}<=0.3
$$


197 existence of significant geometric IR color differences in the injection area was 198 classified as a negative result.

199 Statistics results were made by means of SPSS soft. Parametric and non 200 parametric tests were applied to observe the differences between groups. A Bland201 Altman plot and the Kappa Statistic were also applied to see the inter-observer variation. 


\section{RESULTS}

Table 1 expresses the anthropometric characteristics of 21 subjects $(7 / 14 \mathrm{M} / \mathrm{F})$ suspected of tuberculosis contact. Mean age was 57(17.9) years. Six had a positive PPD lecture (more than $5 \mathrm{~mm}$ of papule). In 15 subjects IR analysis was positive for tuberculine test.

Positive reaction of the IR visual examination was identified as one or more central geometric images with circular, elliptic or with more irregular contours, easy to differentiate from the rest of the IR surface (Figure 1). A higher temperature of this area appeared with a more intense color respect to the neighboring (blue to red colour scale). In subjects with a negative PPD test these characteristics were not evident in the IR image. Only one subject with negative PPD reaction presented a IR positive characteristic image.

Table 2 depicts IR parameters for the two groups of subjects: Group 1 with a positive IR image (15 subjects) and Group 2 with negative IR reaction (6 subjects). Mean, min, and maximum temperatures were higher for group 1 than group 2. Differences for mean temperature were around $1{ }^{\circ} \mathrm{C}$ between both groups. These parameters were higher in area 1 than area 2 for group 1 and there were not significant differences from group 2. Differences for group 1 were around $0.5^{\circ} \mathrm{C}$ between area 1 and area 2.

Regression coefficient between diameter of area 1 measured by exploration and IR analysis was $\mathrm{R}=0.8, \mathrm{R}^{2}=0.64$, constant $=6.13$, coefficient beta=0.8.p $<0.001$. for all subjects with a positive PPD (14). Mean difference of diameter measured by PPD examination and IR analysis was around $1.4 \mathrm{~mm}(-12.6$ to 5.5$)$. 
A Bland-Altman plot compares two assay methods. It plots the difference 228 between the two measurements on the $Y$ axis, and the average of the two measurements on the $X$ axis (figure 4). Differences between diameter of induration measured by exploration of PPD and IR image analysis of area 1 were into the $95 \%$ limits of agreement (mean bias plus or minus 1.96 times its SD). The bias is computed as the value determined by one method minus the value determined by the other method. Bias was 1.4. and SD of Bias was 5.25. 95\% limits of agreement were from 8.9 to 11.7. Inter-observer variation was measured by The Kappa Statistic, between exploratory lecture of PPD and IR image analysis (Table 3). The agreement was 0.89 (almost perfect 0.81-0.99. Kappa Index).

\section{DISCUSSION}

The authors studied the skin tuberculine reaction in 21 tuberculosis contacts by

infrared emission image analysis and compared it with classical tuberculine examination.

242 Fourteen subjects with a tuberculin reaction size more than $5 \mathrm{~mm}$ had an IR positive 243 image reaction. Six subjects with negative tuberculin test had also a negative IR image.

244 One contact with a negative tuberculin test had a positive IR image. The diameter of 245 the IR reaction was higher than the diameter measured by skin examination. Infrared imaging has been extensity applied in medicine since the 1960's.

247 Detection of peripheral vascular disorders and breast cancer are some 248 examples $7,14,15,16,17$. In areas affected by a inflammation process characterized by 249 arteriolar capilar and venula dilatation with inflammatory infiltrate there is an increase 250 in an increasing of eat ${ }^{10}$. IR is able to detect skin temperature differences as low as 0.1 
$251 \mathrm{C}^{\mathrm{o} 18}$. These properties had been applied in several medical cases as control of bar 252 infection ${ }^{19}$, replacement of total $\mathrm{knee}^{20}$ and acne treatments ${ }^{21,22}$.

253

The current measurement of tuberculine reaction is the subjective measure of 254 skin induration by tact. However, the reading of induration could be a potential error 255 source ${ }^{5}$. Margins of the induration are difficult to find independently of technique 256 (ballpoint-pen, palpation) $)^{4}$. On the other hand, it could be a subcutaneous inflammation 257 due to the tuberculine reaction but not evident by palpation as a false negative result or 258 doubtful lecture ${ }^{4}$. In this case, skin IR emission could be more sensible that a simple 259 examination. Histopathologic pattern of human intracutaneous tuberculine reaction was 260 described by Kuramoto ${ }^{23}$ and more recently by Haholu ${ }^{11}$. These reactions take place in 261 the epidermis and dermis. These cellular infiltrations with an extensive vascular 262 component (vasodilatation, edema exudation) are responsible for an increase in the heat 263 generation on the injection place that can be measured by IR camera ${ }^{10}$. Changes from 2640.2 to $2{ }^{\circ} \mathrm{C}$ have been demonstrated in inflammatory dermatologic processes ${ }^{20,24,25}$.

We observed a significant difference in the mean temperature between the 266 central segmented IR area and window hand area at around $1 \mathrm{C}^{\mathrm{o}}$. This temperature was 267 above that measured in healthy subjects ${ }^{8}$. In addition, the difference between the central 268 and surrounding area temperatures was significant, around $0.5 \mathrm{C}^{\circ}$. A higher production 269 of heat indicates different inflammatory events in the same way that the small 270 indurations tact is different from the skin eritema area. Infection with $\mathrm{M}$ tuberculosis 271 causes a cell-mediated immune response leading to sensibilized $\mathrm{T}$ lymphocyte ${ }^{26}$. 272 Intradermal injection of PPD evokes a delayed hyper sensibility response mediated by 273 sensitized $\mathrm{T}$ cells that produces a skin induration ${ }^{23}$. Erithema multiform and spongiotic 274 dermatitis are more related with PPD score based on the induration area. The most 
275 frequent, basal spongiotic dermatitis, is characterized by edematous changes in the 276 epidermis and mononuclear exocitosys. In addition, the erythema multiform type has an 277 important component of edema with erythrocyte extravasations. In consequence we 278 believe that marked edema as a consequence of a prolonged vasodilatation with cellular 279 extravasations are responsible for increased temperature in the central area. This fact is 280 shown by an increase of irradiated heat as observed in other inflammatory diseases ${ }^{10}$.

One important finding of the current work was that one subject with a negative 282 PPD had a positive reaction to the IR analysis. The possibility that PPD shows false negatives has been previously reported ${ }^{27}$. An immune compromised state (HIV infection) or inmunologic-suppression and booster phenomenon may lead to false negative results $^{27}$. Methodological technical causes such as sub-dermic, superficial injection with an easy rupture of the vesicle, as well as a subjective interpretation caused by inter287 reader variability could constitute other causes of false negative results ${ }^{5}$. In fact, there is 288 a $10 \%$ rate of false negative PPD's in hospitalized patients, increasing to $50 \%$ in cases 289 of disseminate tuberculosis ${ }^{26}$.In consequence, IR could substantially improve the sensibility in these cases. We are not able to add any new data regarding false positive 291 cases, which is an intrinsic problem of the tuberculine reaction. IR only provides 292 information about local skin inflammatory reactions regardless of the kind of 293 inflammation or germ-causing disease. New immune based tests such as interferon- $Y$ 294 release assay or POC technologies could increase the sensibility and specificity of 295 tuberculosis diagnostic ${ }^{6}$.

Several issues related to methodology of the present paper must be addressed.

297 First, the reduced group of patients has not permitted us to draw general conclusions, 298 but in the present work a positive IR reaction was observed in all TST manual lecture 
299

300

301

302

303

304

305

306

307

308

309

310

311

312

313

314

315

316

317

318

319

320

except in one and also negative IR reaction was observed in all negative TST test. In conclusion, although it needs a larger sample to extract definitive conclusion, we thing that these results induce to perform a multicentre study that will permit the definitive generalization of present procedure. On the other hand, infrared reading was made following the guidelines for standardization in Medical Thermography ${ }^{17}$. We think that this procedure could applied with no restrictive conditions that will permit to use more simple elements as an IR mobile-camera in different spaces for its general use.

Worth to mention that our previous work $^{28}$ revealed that thermal imaging provides complementary information to visual imaging, from an information theory point of view.

\section{Conclusion}

IR may represent an improved estimation of tuberculosis infection, given that, IR lecture does not depend on reader variability and only measures the increase of heat irradiation produced by the allergic tuberculin response, which is the IR physical principle that could be objectively measured.

\section{REFERENCES}

1. WHO. Report 2004. Global tuberculosis control. Survillance. Planning. Financing. WHO edit. Ginebra 2004.

2. Gallardo J, Ramos A, Jara B, Ancochea J. Tuberculosis. In Manual de Neumología Clínica (2º edition). Diez JM, Alvarez-Sala R editors (ERGON). 2009 193:213 
3. American Thoracic Society and Centers for Disease Control And Prevention: Diagnostic standars and classification of tuberculosis in adults and children. Am J Respir Crit Care Med 2000 161:1376.

4. Bouros D, Zeros G, Panaretos C, Vassilatos C, Siafakas N. Palpation vs pen method for the measurement of skin tuberculin reaction (Mantoux test). Chest. 1991 99:416-9.

5. Pouchot J, Grosland A, Collet C. Coste J, Esdaile JM, Vinceneux P. Reliability of tuberculin skin test measurement. Ann Intern Med 1997 126:210-4.

6. Pai M, Minion J, Sohn H, Zwerling A, Perkins M.D. New technologies for tuberculosis diagnosis. Clin Chest Med 2009 30:701-716.

7. Bagavathiappan S, Sarananan T, Philip J, Jayakumar T Ph J, Baldev R, Karunanithi R, Panicker T.M.R, Korath M.P, Jagadeesan K. Infrared thermal imaging for detection of peripheral vascular disorders. J Med Phys 2009 34:4347.

8. Ring EFJ, Ammer K. The technique of infrared imaging in medicine. Thermology International 2000 1:7-14.

9. Schaefer A.L, Cool N, Tessaro S.V, Deregt D, Desroches G, Dubeski P.L, Tong A.K.W, Godson D.L. Early detection and prediction of infection using infrared thermography. Can J Anim Sci 2004 84:73-80.

10. Ring F.E, Ammer K, Thermal imaging in diseases of the skeletal and neuromuscular systems. In Medical infrared imaging. Edited by N.A. Dinkides, J.D. Bronzino CRC, US. 2008:17-1.

11. Haholu A, Ciftci F, Karabudak O, Kutlu A, Bozkurt B, Baloglu H. The significance of histopathologic patterns in positive tuberculin skin test. Journal of Cutaneous Pathology. 2008 35:462-465.

12. De Souza-Galvao M.L, Latorre I, Altet-Gómez N, Jiménez_Fuentes, Milà C, Solsona J, Seminario M.A, Cantos A, Ruiz-Manzano J, Dominguez J. 
Correlation between tuberculine skin test and IGRAs with risk factors for the spread of infection in close contacts with sputum smear positive in pulmonary tuberculosis. BMC. Infectious Diseases 2014 14:258.

13. Gonzales R. C., Woods R. E. Digital Image Processing. New Jersey: Prentice Hall; 2002.

14. Wang J, Chang KJ, Chen CY,Chien KL, Tsai YS, Wu YM, Teng YC, Shiht T. Evaluation of the diagnostic performance of infrared imaging of the breast: a preliminary study. BioMedical Engineering Online 2010 9:3.

15. Ring EFJ. Standardization of Thermal Imaging in Medicine: Physical and Environmental factors in Thermal Assessment of Breast Health. Ed Gautherie M, Albert E, Keith L, 29-36. MTP Press Ltd. Lancaster/Boston/The Hague. 1983.

16. Engel JM, Cosh JA, Ring EFJ, Page DP, Van Waes P, Shoenfeld D. Thermography in Locomotor Diseases- Recommended Procedure. Eur J Rheum Inflamm 1979 2:299-306.

17. Clark RP, de Calcina-Goff M. Guidelines for Standardization in Medical Thermography Draft International Standard Proposals. Thermologie Österreich 1997 7:47-58.

18. Cetingül M, Herman C. Identification of subsurface structures from the transiet thermal response and surface temperature measurements, in: Proceedings of the $5^{\text {th }}$ European Thermal-Sciences Conference 18-22 May, Eindhoven 2008: 1219-1222.

19. Fujita K, Noguchi M, Yuzurika Sh, Yanagisama D, Matsuo K. Usefulness of infrared thermal imaging camera for screening of postoperative surgical site infection after the noss procedure. Case Rep Surg 2013:946156. 
20. Romariò CL, Logoluso N, Dell'Oro F, Elia A, Drago L. Telethermographic findings after uncomplicated and septic total knee replacement. Knee 2012 19:193-7.

21. Padilla-Medina JA, León-Ordoñez F, Prado-Olivarez J, Vela-Aguirre N, Ramirez-Agundis A, Diaz-Carmona J. Assessment technique for acne treatments based on statistical parameters of skin thermal images. Comput Biol Med 2004 47:36-43.

22. Herman C. The role of dynamic infrared imaging in melanoma diagnosis. Expert Rev Dermatol 2013 8:177-184.

23. Kuramoto Y, Tagani H. Histopathologic pattern analysis of human intracutaneous tuberculin reaction. The American Journal of Dermathopathology 1989 11:329-337.

24. Van Netten JJ, Van Baal JG, Liu C, Cn der Jeijden F, Bus SA. INfrared thermal imaging for automated detection of diabetic foot complications. $\mathbf{J}$ Diabetes Sci Technol 2013 1:1122-9.

25. Han SS, Jung CH, Lee SC, Jung HJ, Kim YH. Does skin temperature difference as measured by infrared thermography within 6 months of acute herpes zoster infection correlate with pain level?.

26. Brodie D, Schluger N.W. The diagnosis of tuberculosis. Clinics in chest medicine 2005 26:247-271.

27. Huebner RE, Schein MF, Bass Jr JB. The tuberculin skin test. Clin Infect Dis 1993 17:968-75.

28. Virginia Espinosa-Duró, Marcos Faundez-Zanuy, Jiri Mekyska, Enric MonteMoreno. A Criterion for Analysis of Different Sensor Combinations with an Application to Face Biometrics. Cogn Comput (2010) 2:135-141 
400 TABLE 1 ANTROPHOMETRIC CHARACTERISTICS OF SUBJECTS WITH

401

SUSPECTED TBC

402

403

Male

Female

(M/F) Age(ys) Height(cm) Weight(Kg) Age(ys) Height(cm) Weight(Kg) Total

All

$\begin{array}{lllllll}57.0(17.8) & 156.0(17.4) & 70.0(20.1) & 51.4(15.9) & 155.8(8.1) & 52(11.6) & 21\end{array}$

$\begin{array}{llllllll}\text { Positive } & 48.3(18.7) & 172.5(5.9) & 73.1(10.0) & 45.2(13.8) & 159.3(6.4) & 64.9(8.7) & 15\end{array}$

$(6 / 9)$

$\begin{array}{llllllll}\text { Negative } & 32 & 189 & 97 & 62.4(14.3) & 149.4(7.3) & 64.7(16.9) & 6\end{array}$

$(1 / 5)$

404

405

406

Table 1. Anthropometric values of 21 subjects with tuberculosis contact 407 suspected. Positive and negative test responses were measured by infrared analysis (IR)

408 with a term graphic IR camera. All a values are in mean(sd). 
410 TABLE 2. INFRARED LECTURE OF SUBCUTANEAU TUBERCULINE TEST IN 411 PATIENTS WITH SUSPECTED OF TUBERCULOSIS

\begin{tabular}{|c|c|c|c|}
\hline $\begin{array}{c}\text { Group } 1(+) \\
(15)\end{array}$ & Area 1 & Area 2 & Area 3 \\
\hline Area $\mathrm{mm}^{2}$ & $0.96(0.75)^{*}+$ & 6.71 (7.88) & 17.17(7.76) \\
\hline $\mathrm{T} \min \mathrm{oc}^{-}$ & $35.93(0.85)^{*}+$ & $35.33(1.06)$ & 33.52(1.11) \\
\hline $\mathrm{T} \max \stackrel{\circ}{ } \mathrm{C}$ & $36.34(0.78)^{*}+$ & $36.02(0.83)$ & $35,82(1.08)$ \\
\hline $\mathrm{T}$ mean $\mathrm{oc}$ & $36.04(0.33) *+$ & $35.57(0.96)$ & $34,71(0.93)$ \\
\hline Diameter mm & $15.45(7.24)+$ & $37.45(20.53)$ & \\
\hline Group $2(-)$ & Area 1 & Area 2 & Area 3 \\
\hline (6) & & & \\
\hline Area $\mathrm{mm}^{2}$ & 7.54 (5.31) & 5.71 (2.91) & 6.27 (3.59) \\
\hline$T \min { }^{\circ} \mathrm{C}$ & $34.27(3.45)$ & 34.17(1.77) & $32.78(2.00)$ \\
\hline $\mathrm{T} \max \stackrel{\circ}{ }=\mathrm{C}$ & $35.15(1.51)$ & $35.07(1.61)$ & $35.73(1.46)$ \\
\hline T mean oc & $35.18(1.68)$ & $34.51(1.67)$ & $34.58(1.48)$ \\
\hline
\end{tabular}

- * Signification level $\mathrm{p}<0.05$. Non parametric U Mann-Whitney Test between Groups

- $\quad+$ Signification level $\mathrm{p}<0.05$. Difference between area 1 and 2. Non parametric Wilcoxon Rang-sig test for the same group.

Group 1:Tuberculine lecture IR positive ; Group 2: Tuberculine lecture IR negative. 
TABLE 3. INTEROBSERVER AGREEMENT. TheKappa Statistic

425

426

\begin{tabular}{||l|l|c|c|c|}
\hline \multicolumn{2}{|c|}{} & \multicolumn{3}{|c|}{ Exploratory lecture of PPD } \\
\cline { 2 - 5 } & Positive & Negative & Totals \\
\hline \multirow{3}{*}{ IR image } & Positive & 14 & 1 & 15 \\
\cline { 2 - 6 } & Negative & 0 & 6 & 16 \\
\cline { 2 - 6 } & Totals & 14 & 7 & 21 \\
& & & & \\
\hline
\end{tabular}

427

428 Kappa Statistic agreement between observed PPD exploration and IR image

429 analysis in 21 subjects with TBC contact suspect.

430 Absolute agreement $=0.95$. Hope agreement $=0.57$.

431 Kappa Index: 0.89. Standar error I.C 95\% (0.67-1.1). 


\section{FIGURE 1-IR images in a positive PPD test.}

Figure 1-1 is the photograph of the injection tuberculine area. We can observe a

435 higher IR intensity in the area of tuberculine injection (Figures 1-1,1-3). Figure 1-2 is

436 a superposition of IR and colour photographic image. To have a reference distance, we 437 placed a $24 \mathrm{~mm}$ diameter coin below the area to be measured

FIGURE 2 - Flowchart of the IR image processing algorithm.

First, image temp was scaled, thus obtaining image temp_n. Then, the reference

441 object was detected (center and radius), which allowed us to calculate the conversion

442 factor from pixels to millimeters. Next, an intensity transformation was applied to 443 temp_n, thus obtaining image temp_e. A region growing segmentation algorithm was 444 applied to temp_e in order to obtain region 1, region 2, and the background. Finally, 445 some parameters are calculated for each region and these parameters were used to 446 decide if some region were merged or not.

\section{FIGURE 3-Different aspects of the image processing algorithm}

(a) temp_n image; (b) Edges detection by Canny's method; (c) temp_n image after 450 rejecting the internal pixels of the reference object; (d) temp_e image (white expansion) 451 and the analysis area (red square); (e) Analysis area mask; (f) PPD reaction area (white), 452 erythema (light gray), and background (dark gray); (g) PPD reaction area and its 453 maximum distance; (h) Erythema and its maximum distance; (i) Edges of the PPD 454 reaction area and the erythema. 
456 FIGURE 4. Bland-Altman plot

457

458 A Bland-Altman plot compares two assay methods. It plots the difference

459 between the two measurements on the $\mathrm{Y}$ axis, and the average of the two measurements

460 on the $\mathrm{X}$ axis. In this case the two measures are: the diameter measured by manual

461 exploration of the PPD papula and the diameter of area 1 measured by IR image

462 analysis. The $95 \%$ limits of agreement are shown as two dotted lines.

463 
1

2

3

4

5
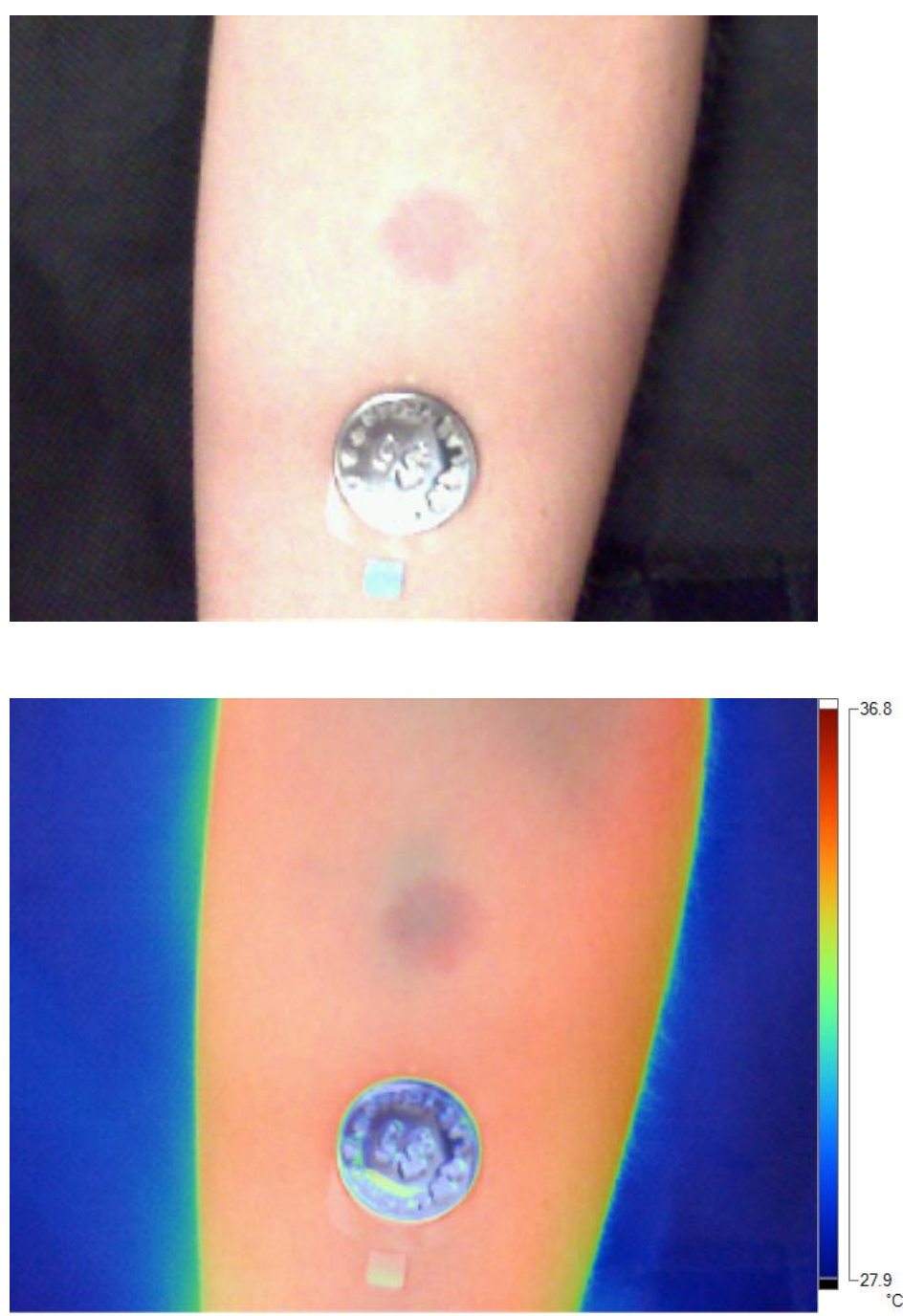

467

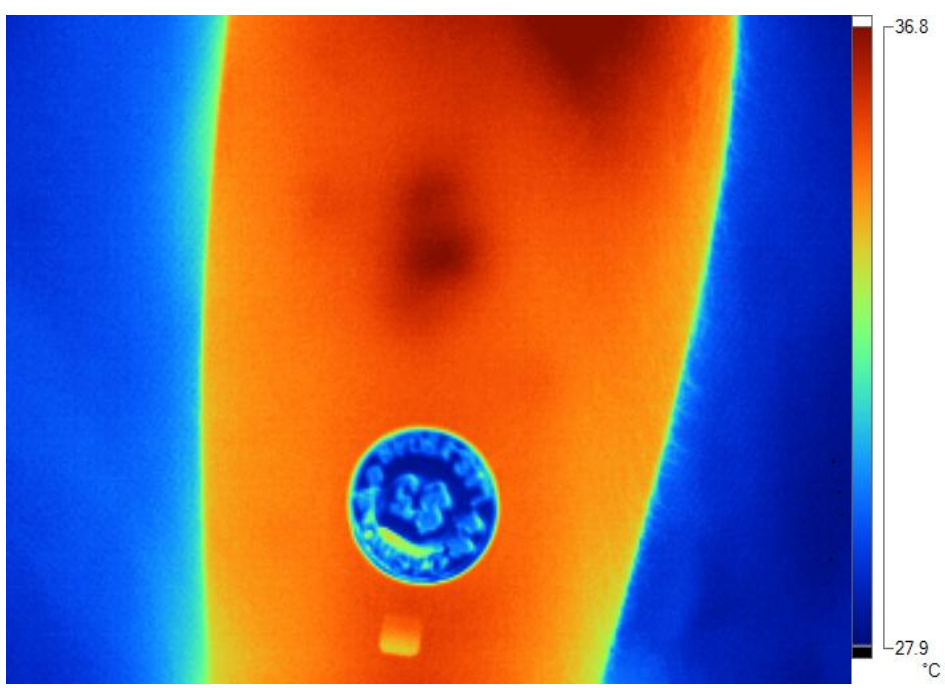

468 FIGURE 1.IR images in a subject with a positive PPD test 
Radius of the circular reference abject $\{\mathrm{mm}\}$

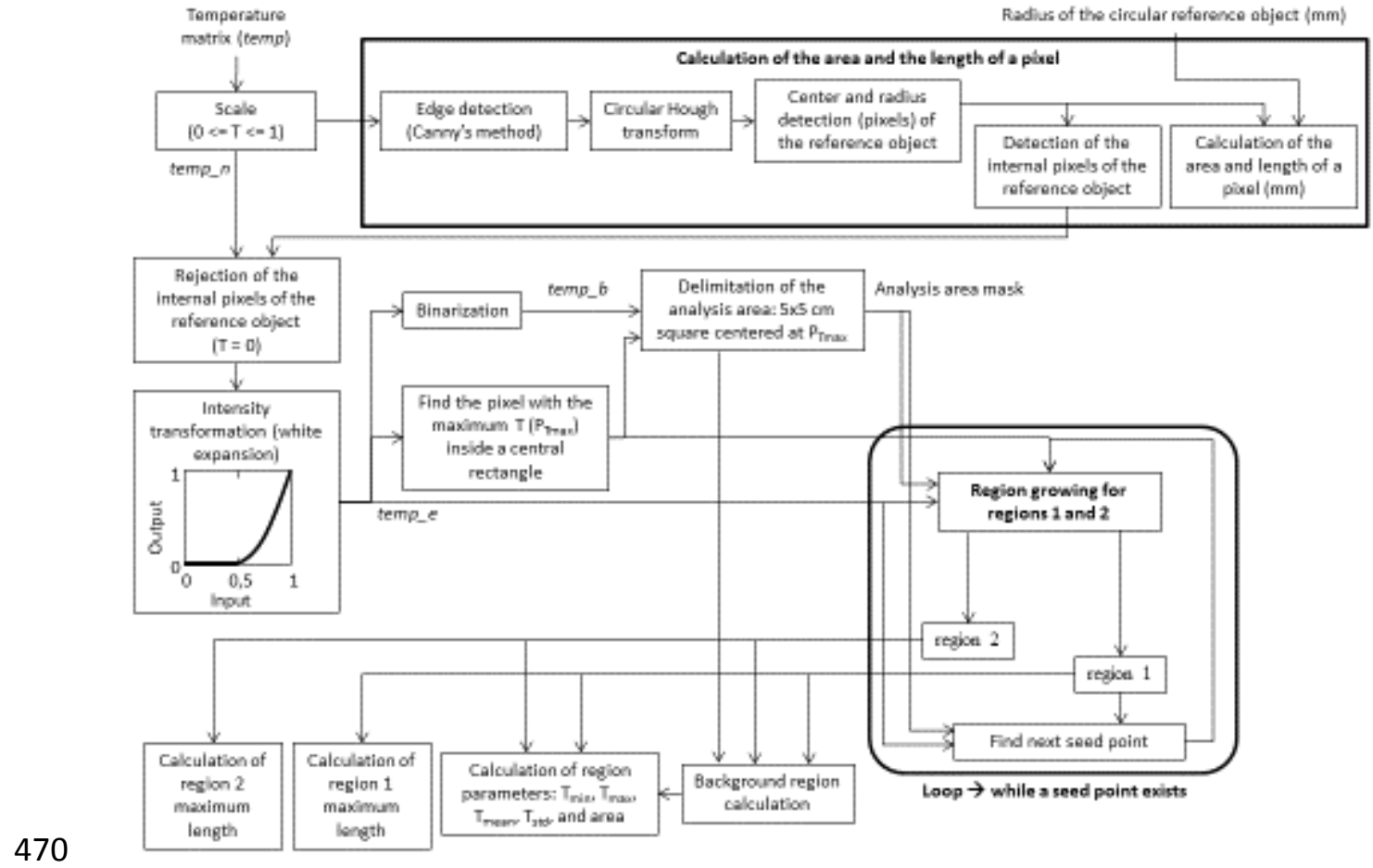

471

472

FIGURE 2 . Flowchart of the IR image processing algorithm

473

474

475

476

477

478

479

480

481

482

483

484

485

486

487

488

489

490 


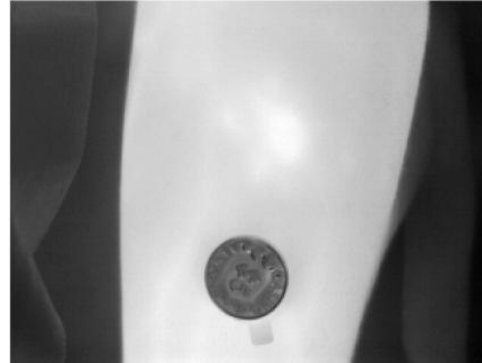

(a)

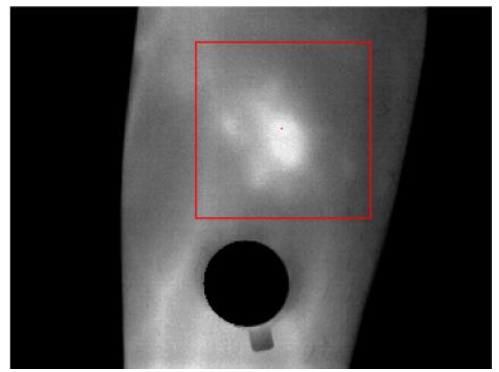

(d)

493
494

495

496

497

498

499

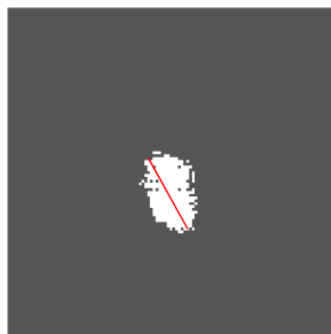

(g)

\section{algorithm}

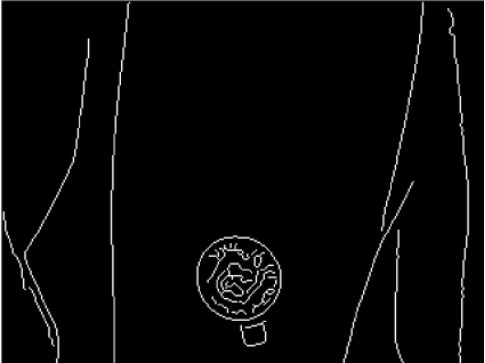

(b)
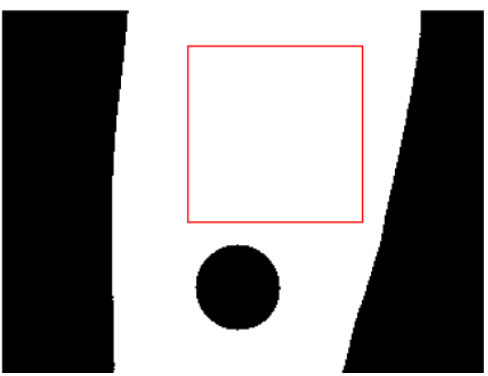

(e)

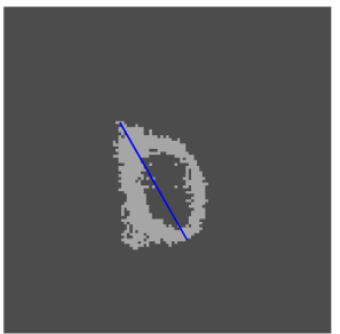

(h)

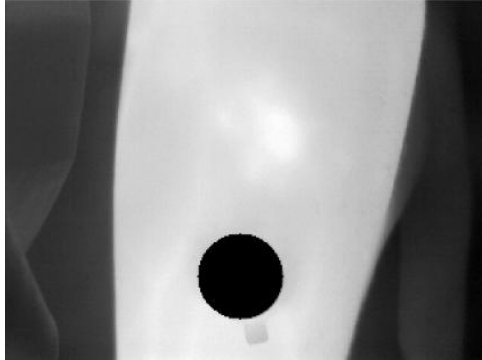

(c)

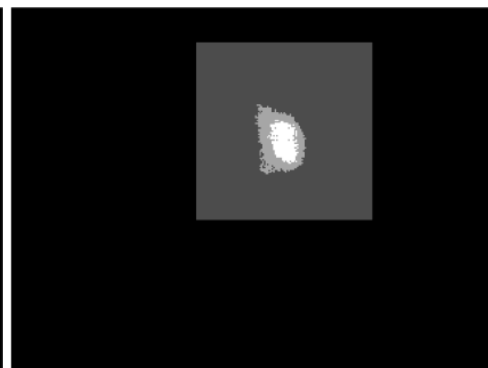

(f)

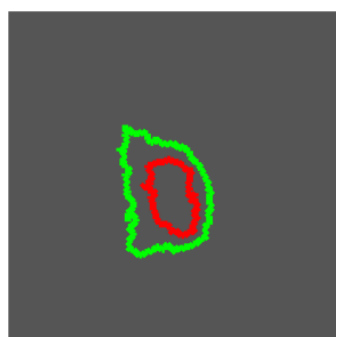

(i)

FIGURE 3. Different aspects of the image processing

500

501

502 
503

504

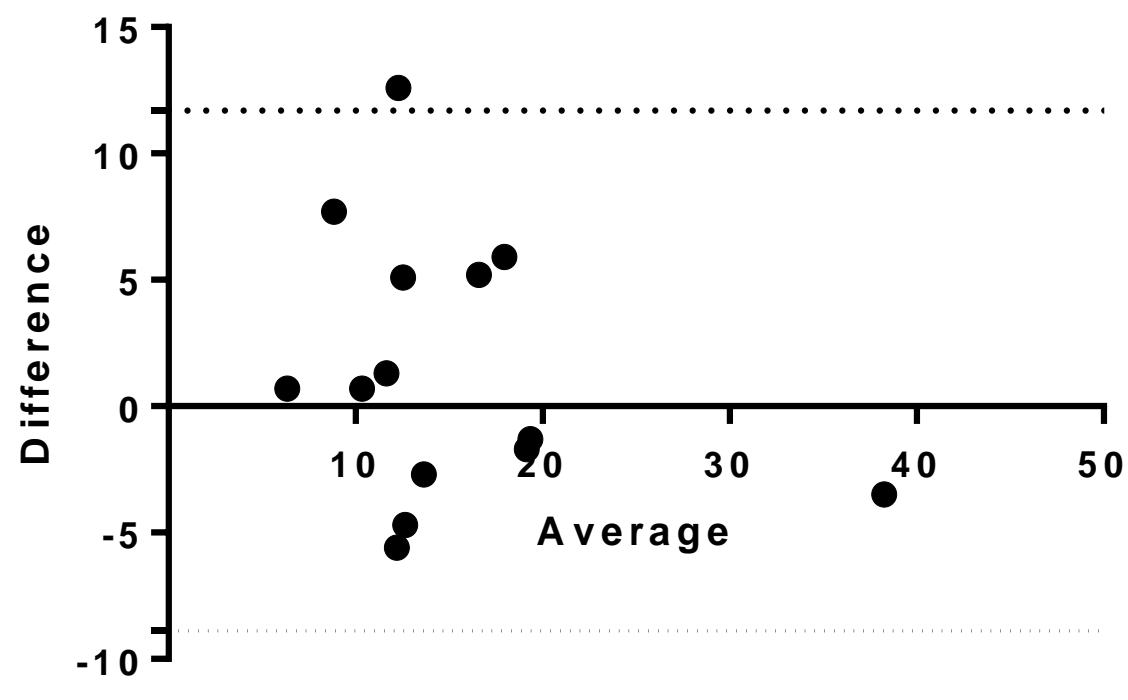




\section{Declaration of conflict of interest}

The authors state that they have no conflict of interest in the elaboration of this paper.

\section{Acknowledgements}

The authors wish to thank the nurses Rosa Gomez and Aurora Torrents for their special assistance.

\section{AUTHOR'S CONTRIBUTIONS:}

- José Antonio Fiz Fernández: inventor of procedure, design of the work; processed and automation of image analysis; analysis and interpretation of the data for the work; writing the article; guarantor of the paper.

- Manuel Lozano :Processed and automation of image analysis; analysis and interpretation of the data for the work; writing the article

- Enrique Monte Moreno: Analysis and interpretation of the data; writing the article; revision of the word.

- Marcos Faundez: IR support and revision of the word.

- Laura Rodriguez Pons: Clinical study of patient Inclusion

- Caroline Becker: Clinical study of patient Inclusion.

- Adela Gonzalez_Martinez: PPD test realization.

- Juan Ruiz Manzano. :Clinical study of patient Inclusion. 